Internat. J. Math. \& Math. Sci.

Vol. 24, No. 3 (2000) 179-185

S016117120001005X

(C) Hindawi Publishing Corp.

\title{
SINGULAR POINTS AND LIE ROTATED VECTOR FIELDS
}

\author{
JIE WANG and CHEN CHEN
}

(Received 2 April 1999)

\begin{abstract}
This paper gives the definition of Lie rotated vector fields in the plane and the conditions of movement of singular points on Lie rotated vector fields with variable parameters.
\end{abstract}

Keywords and phrases. Lie rotated vector fields, Lie bracket, one parameter group, singular points.

2000 Mathematics Subject Classification. Primary 34C05.

1. Introduction. Many engineering problems are usually run into a class of nonlinear equations that contain variable parameters. In order to study whole orbits or whole phase diagrams of vector fields that contain parameters, it is a complicated and interesting problem how the whole orbit or whole phase diagram change as parameter is changed. It is extremely complicated for general containing parameter vector fields to change in the plane, but for some special containing parameter rotated vector fields, their change has regular rule as parameter is changed. These are many results in this respects [3, 4, 5, 6, 7].

In Section 2, we present the basic definitions of Lie rotated vector fields. We define Lie rotated vector fields using one parameter group approach. In accordance with the strict definition of rotated vector field, the singular points of $X(\mu)$ must be kept fixed, but in this paper, the singular points of $X(\mu)$ can be moved as parameter $\mu$ is changed. In Section 3, we discuss the motion of singular points on Lie rotated vector fields. In the section, we require the singular points of $X(\mu)$ to be strictly moved as parameter $\mu$ is changed, and permit the moved singular points to disappear or decompose, which do not coincide with the singular points of original vector field. We give some conditions and properties corresponding to the vector field $Y$. In this paper, we give some examples to illustrate the concept and notion of Lie rotated vector fields.

2. Lie rotated vector fields. We consider vector fields on the plane $x=\left(x_{1}, x_{2}\right)$ $\in \mathbb{R}^{2}$,

$$
X=\left(X_{1}(x), X_{2}(x)\right), \quad Y=\left(Y_{1}(x), Y_{2}(x)\right) .
$$

For the vector fields (2.1), we define

$$
X \wedge Y=X_{1} Y_{2}-X_{2} Y_{1}, \quad\langle X, Y\rangle=X_{1} Y_{1}+X_{2} Y_{2}
$$

If $X$ and $Y$ are vector fields, then $[X, Y]$ is a vector field which is operated by Lie 
bracket, i.e.,

$$
[X, Y]=\left(Z_{1}, Z_{2}\right),
$$

where $Z_{1}$ and $Z_{2}$ are expressed as,

$$
Z_{1}=\left\langle X, \nabla Y_{1}\right\rangle-\left\langle Y, \nabla X_{1}\right\rangle, \quad Z_{2}=\left\langle X, \nabla Y_{2}\right\rangle-\left\langle Y, \nabla X_{2}\right\rangle
$$

respectively, where $\nabla$ is gradient operator.

Let the plane vector fields $X(\mu)=\left(X_{1}(x, \mu), X_{2}(x, \mu)\right)$ be defined by the following differential equations:

$$
\frac{d x_{1}}{d t}=X_{1}(x, \mu), \quad \frac{d x_{2}}{d t}=X_{2}(x, \mu),
$$

where $X_{1}$ and $X_{2}$ are functions of $x$ and parameter $\mu \in I \subset \mathbb{R}$, and the singular points are isolated.

DEFINITION 2.1. Let the plane vector field $X(\mu)$ be determined by (2.5), where $X_{1}, X_{2} \in C^{3}\left(\mathbb{R}^{2} \times I, \mathbb{R}\right), I=\{\mu|| \mu \mid<\delta\}$ is a real interval, $\delta$ is a given positive number. If vector field $Y$ exists which is defined by the following differential equations:

$$
\frac{d x_{1}}{d t}=Y_{1}(x), \quad \frac{d x_{2}}{d t}=Y_{2}(x),
$$

where $Y_{1}$ and $Y_{2} \in C^{3}\left(\mathbb{R}^{2}, \mathbb{R}\right)$. At all ordinary points of $X(0)$, such that the following relation holds

$$
L(0) \stackrel{\text { def }}{=} X(0) \wedge\left\{X_{\mu}^{\prime}(0)+[X(0), Y]\right\}>0(<0),
$$

where $X_{\mu}^{\prime}(0)$ is the derivative of the vector field $X(\mu)$ at $\mu=0$, then $X(\mu), \mu \in I$, is called Lie rotated vector fields.

REMARK 2.2. If the vector field $X(\mu)$ is defined on $D \times I$, where $D \subset \mathbb{R}^{2}$, such that $X(0)$ satisfies relation (2.7) at all ordinary points of $X(0)$ on $D$, then $X(\mu), \mu \in I$, is called Lie rotated vector fields on $D$.

LEMMA 2.3. Let $\psi^{s}$ be a one parameter transform group which is produced by $C^{1}$ vector field $Y, s \in \mathbb{R}$, and let $X$ be $C^{1}$ vector field. If $s$ is fixed, and $\varphi_{p}(t)$ is an integral curve of $X$ through the point $p, \varphi_{p}(0)=p$, then $\psi^{s} \circ \varphi_{p}(t)$ is an integral curve of $\psi_{*}^{s} X$ through the point $\psi^{s}(p)$. If $\left.X\right|_{p}=0$, then $\left.\left(\psi_{*}^{s} X\right)\right|_{\psi^{s}(p)}=0$.

Proof. The proof follows from [1] and [2]. In fact, if $\varphi_{p}(t)$ is an integral curve of $X$ through the point $p$, then

$$
\left.\left(\psi^{s} \circ \varphi_{p}(t)\right)\right|_{t=0}=\psi^{s}(p)
$$

and

$$
\begin{aligned}
& \left(\psi^{s} \circ \varphi_{p}(t)\right)_{*_{t}} \cdot\left(\left.\frac{d}{d t}\right|_{t}\right)=\psi_{*_{\varphi_{p}(t)}^{s}} \circ \varphi_{p(t)_{*_{t}}} \cdot\left(\left.\frac{d}{d t}\right|_{t}\right) \\
& =\psi_{*_{\varphi_{p}(t)}^{s}}^{s} \cdot X_{\varphi_{p}(t)}=\left(\psi_{*}^{s} X\right)_{\psi^{s} \circ \varphi_{p}(t)} .
\end{aligned}
$$


It follows that $\psi^{s} \circ \varphi_{p}(t)$ is an integral curve of $\psi_{*}^{s} X$ through the point $\psi^{s}(p)$.

Next, due to

$$
\left.\psi_{*}^{s} X\right|_{q}=D \psi^{s}\left(\psi^{-s}(q)\right) \cdot X\left(\psi^{-s}(q)\right), \quad q \in \mathbb{R}^{2} .
$$

Set $q=\psi^{s}(p)$, note that we already suppose $\left.X\right|_{p}=0$, again note that $\psi^{s}$ is a one parameter transform which is produced by $Y$, then

$$
\left.\psi_{*}^{s} X\right|_{\psi^{s}(p)}=D \psi^{s}(p) \cdot X(p)=\left.D \psi^{s}(p) \cdot X\right|_{p}=0,
$$

i.e., $\psi^{s}(p)$ is a singular point of $\psi_{*}^{s} X$.

LEMMA 2.4. Let $\psi^{s}$ be a one parameter transform group which is produced by $C^{1}$ vector field $Y, s \in \mathbb{R}$, fix $s$, then the index of isolated singularity of $C^{1}$ vector field $X$ is not changed under the $\psi^{s}$ transform.

Proof. In fact, by the condition of the lemma, it is known that $\psi^{s}$ is a differentiable homeomorphism, then the lemma follows from [8, Theorem 4.2].

Next, if $X(\mu)$ is a Lie rotated vector field, then $Y$ is a corresponding vector field which satisfies (2.7), and $\psi^{s}$ is a one parameter transform group which is produced by $Y, s \in \mathbb{R}$.

LEMMA 2.5. Let $X(\mu)$ be a Lie rotated vector field, for all $\varepsilon>0$, there exist $\delta=\delta(\varepsilon)$, such that when $|\mu|<\delta, \psi_{*}^{s} X(\mu)$ constitutes a rotated vector field.

Proof. Let the singular points of $\psi_{*}^{s} X(\mu), \mu \neq 0$, on the plane $\mathbb{R}^{2}$ be $p_{\mu_{1}}, \ldots, p_{\mu_{k}}$ and the singular points of $X(0)$ on the plane $\mathbb{R}^{2}$ are $p_{1}, \ldots, p_{m}, \forall \varepsilon>0,0<\varepsilon \ll 1$, let $S_{\varepsilon}\left(p_{\mu_{i}}\right)$ or $S_{\varepsilon}\left(p_{j}\right)(1 \leq i \leq k, 1 \leq j \leq m)$ be open neighborhood $p_{\mu_{i}}(1 \leq i \leq k)$ and $p_{j}(1 \leq j \leq m)$, and radius $\varepsilon$, such that $\overline{S_{\varepsilon}(p)} \cap \overline{S_{\varepsilon}(q)}=\varnothing$, where $p$ and $q \in\left\{p_{\mu_{i}}\right\} \cup\left\{p_{j}\right\}$ $(1 \leq i \leq k, 1 \leq j \leq m), p \neq q$. Let $\psi^{s}$ be a one parameter transform group which is produced by $C^{1}$ vector field $Y, s \in \mathbb{R}$. By the limit definition of Lie bracket, we have

$$
\begin{aligned}
\psi_{*}^{s} X(\mu) & =\psi_{*}^{0} X(\mu)+\left.\frac{s}{1 !} \frac{d}{d t}\right|_{s=0} \psi_{*}^{s} X(\mu)+\left.\frac{s^{2}}{2 !} \frac{d^{2}}{d t^{2}}\right|_{s=0} \psi_{*}^{s} X(\mu)+\cdots \\
& =X(\mu)+\frac{s}{1 !}[X(\mu), Y]+\frac{s^{2}}{2 !}[[X(\mu), Y], Y]+\cdots .
\end{aligned}
$$

Next, we notice that $X(\mu)$ can be unfolded as

$$
X(\mu)=X(0)+\frac{\mu}{1 !} X_{\mu}^{\prime}(0)+\frac{\mu^{2}}{2 !} X_{\mu}^{\prime \prime}(0)+\cdots,
$$

since

$$
[X(\mu), Y]=[X(0), Y]+\frac{\mu}{1 !}\left[X_{\mu}^{\prime}(0), Y\right]+\frac{\mu^{2}}{2 !}\left[X_{\mu}^{\prime \prime}(0), Y\right]+\cdots .
$$

Let $s=\mu$, it follows from (2.12), (2.13), and (2.14) that

$$
\begin{aligned}
\psi_{*}^{\mu} X(\mu)= & X(0)+\mu\left\{X_{\mu}^{\prime}(0)+[X(0), Y]\right\} \\
& +\frac{1}{2} \mu^{2}\left\{X_{\mu}^{\prime \prime}(0)+2\left[X_{\mu}^{\prime}(0), Y\right]+[[X(0), Y], Y]\right\}+\cdots .
\end{aligned}
$$


At the ordinary points of $\mathbb{R}^{2} \backslash\left\{\bigcup_{i=1}^{k} S_{\varepsilon}\left(p_{\mu_{i}}\right)\right\} \bigcup\left\{\bigcup_{j=1}^{m} S_{\varepsilon}\left(p_{j}\right)\right\}$, for given $\varepsilon>0$, we sooner or later can find $\delta_{1}=\delta_{1}(\varepsilon)>0$, such that when $|\mu|<\delta_{1}$, we have

$$
\begin{aligned}
\psi_{*}^{\mu} X(\mu) \wedge \frac{\partial}{\partial \mu}\left\{\psi_{*}^{\mu} X(\mu)\right\}= & X(0) \wedge\left\{X_{\mu}^{\prime}(0)+[X(0), Y]\right\} \\
& +\mu X(0) \wedge\left\{X_{\mu}^{\prime \prime}(0)+2\left[X_{\mu}^{\prime}(0), Y\right]+[[X(0), Y], Y]\right\}+\cdots \\
= & L(0)+O(\mu)>0(<0)
\end{aligned}
$$

and let $\vartheta(\mu)$ be the crossing angle of $\psi_{*}^{\mu} X(\mu)$ and the $x_{1}$ axis, for given $\varepsilon>0$, we sooner or later can find $\delta_{2}=\delta_{2}(\varepsilon)$, such that when $|\mu|<\delta_{2}$, at the ordinary points of $\mathbb{R}^{2} \backslash\left\{\bigcup_{i=1}^{k} S_{\varepsilon}\left(p_{\mu_{i}}\right)\right\} \bigcup\left\{\bigcup_{j=1}^{m} S_{\varepsilon}\left(p_{j}\right)\right\}\left(\psi_{*}^{\mu} X(\mu)\right.$ is $X(0)$ when $\mu=0, \vartheta(0)$ is the crossing angle of $X(0)$ and the $x_{1}$ axis), so

$$
0<|\vartheta(\mu)-\vartheta(0)|<\pi
$$

Take $\delta=\min \left\{\delta_{1}, \delta_{2}\right\}$, then when $|\mu|<\delta, \psi_{*}^{\mu} X(\mu)$ constitutes a rotated vector field.

REMARK 2.6. In accordance with the strict definition of rotated vector field, the singular points must be kept fixed, but the singular points of $\psi_{*}^{\mu} X(\mu)$ in Lemma 2.5 can be moved as parameter $\mu$ is changed. In the unmistakable circumstance, when $|\mu|<\delta$, we call $\psi_{*}^{\mu} X(\mu)$ a rotated vector.

In the above lemma, $\delta$ needs not be a quite small positive number, i.e., $0<\delta \ll 1$ need not be set up. For the sake of distinctness, we cite an example to illustrate this equation.

EXAMPLE 2.7. Let $X(\mu)=\left(x_{2},-x_{1}+\mu x_{2}\right)$, if we take $Y=\left(-x_{2} / 2,0\right)$, then at all the ordinary points of $X(0)$, we have

$$
X(0)=\frac{1}{2\left(x_{1}^{2}+x_{2}^{2}\right)}>0
$$

that is, $X(\mu)$ is a Lie rotated vector field.

Now we consider the range of $\mu$, because

$$
\psi_{*}^{\mu} X(\mu)=\left(\frac{1}{2} \mu x_{1}+\left(1-\frac{1}{4} \mu^{2}\right) x_{2},-x_{1}+\frac{1}{2} \mu x_{2}\right)
$$

so

$$
\psi_{*}^{\mu} X(\mu) \wedge \frac{\partial}{\partial \mu}\left\{\psi_{*}^{\mu} X(\mu)\right\}=\frac{1}{2}\left(x_{1}^{2}+x_{2}^{2}\right)-\frac{1}{2} \mu x_{1} x_{2}+\frac{1}{8} \mu^{2} x_{2}^{2} .
$$

Formula (2.16) is compared with formula (2.20), we can find that $O(\mu)$ in formula (2.16) is replaced by $O(\mu)$ in formula (2.20),

$$
O(\mu)=-\frac{1}{2} \mu x_{1} x_{2}+\frac{1}{8} \mu^{2} x_{2}^{2}
$$


yet go a step further calculating, we have

$$
\psi_{*}^{\mu} X(\mu) \wedge \frac{\partial}{\partial \mu}\left\{\psi_{*}^{\mu} X(\mu)\right\}=\frac{1}{2} x_{2}^{2}+\frac{1}{8}\left(\mu x_{2}-2 x_{1}\right)^{2}
$$

which is larger than zero at the ordinary points of $X(0)$ and $\psi_{*}^{\mu} X(\mu)$ for all $\mu \in \mathbb{R}$, but the range of $\mu$ that satisfies formula (2.17) is $|\mu|<4$, thus we take $\delta=4$, when $|\mu|<\delta=4, \psi_{*}^{\mu} X(\mu)$ constitutes rotated vector field.

3. The motion of singular points. Let $X(\mu)$ be a Lie rotated vector field, we require the singular points of $X(\mu)$ to be strictly moved as parameter $\mu$ is changed, and permit the singular points that have been moved disappear or decompose, but require the singular points that have been decomposed to be at most limited in number, which do not coincide with the singular points of the original vector field.

If $p$ is a singular point of $X(\mu)$, we name $J_{\mu}(p)$ for index of singular point $p$ of $X(\mu)$, under the same circumstances, $J_{0}\left(p_{0}\right)$ for index of singular point $p_{0}$ of $X(\mu), J_{\mu_{*}}(q)$ for index of singular point $q$ of $\psi_{*}^{\mu} X(\mu)(\mu \neq 0)$.

THEOREM 3.1. Let $X(\mu)$ be a Lie rotated vector field, $\left.X(0)\right|_{p_{0}}=0$, and let $\left.Y\right|_{p_{0}}=0$. If the singular point $p_{0}$ of $X(0)$ disappears or decomposes as $p_{i}(1 \leq i \leq k)$ in $X(\mu)(\mu \neq$ $0)$, then $J_{0}\left(p_{0}\right)=0$, and $J_{\mu}\left(p_{i}\right)=0(\mu \neq 0,1 \leq i \leq k)$.

Proof. First of all, we prove that $J_{0}\left(p_{0}\right)=0$. In fact, because of $\left.X(\mu)\right|_{p_{0}} \neq 0(\mu \neq 0)$, utilize Lemma 2.3 and condition $\left.Y\right|_{p_{0}}=0$, we know that $\left.\psi_{*}^{\mu} X(\mu)\right|_{p_{0}} \neq 0(\mu \neq 0)$, it follows from Lemma 2.5 , for given $\delta>0$, when $|\mu|<\delta, \psi_{*}^{\mu} X(\mu)$ constitutes a rotated vector field. Take $\eta>0$ as quite small positive number, such that $\overline{S_{\eta}\left(p_{0}\right)}$ does not contain the singular points of $\psi_{*}^{\mu} X(\mu)(\mu \neq 0)$, and only contains the isolate singular point $p_{0}$ of $X(0)$. It is easy to know that $J_{\mu_{*}}\left(p_{0}\right)=0$ about $\partial S_{\eta}\left(p_{0}\right)$. By (2.17) of Lemma 2.5, it follows that $J_{0}\left(p_{0}\right)=0$ when $|\mu|<\delta$.

Using the same method, we prove $J_{\mu_{*}}\left(\psi^{\mu}\left(p_{i}\right)\right)=0(\mu \neq 0,1 \leq i \leq k)$ and by Lemma 2.4, we find $J_{\mu}\left(p_{i}\right)=0(\mu \neq 0,1 \leq i \leq k)$.

COROLLARY 3.2. Let $X(\mu)$ be a Lie rotated vector field, $\left.X(0)\right|_{p_{0}}=0$, if $\left.Y\right|_{p_{0}} \neq 0$, and moved the singular points $p_{i} \neq \psi^{-\mu}\left(p_{0}\right)(\mu \neq 0,1 \leq i \leq k)$, then $J_{0}\left(p_{0}\right)=0$ and $J_{\mu}\left(p_{i}\right)=0(\mu \neq 0,1 \leq i \leq k)$.

Proof. Since $\left.X(0)\right|_{p_{0}}=0$, let the singular point of $X(\mu)(\mu \neq 0)$ disappears or decomposes into $p_{1}, \ldots, p_{k}$ points which do not coincide with singular point $p_{0}$ of $X(0)$, i.e., $\left.X(\mu)\right|_{p_{i}}=0(1 \leq i \leq k)$, yet because of $\left.X(\mu)\right|_{p_{0}} \neq 0(\mu \neq 0)$ and $\left.Y\right|_{p_{0}} \neq 0$. By Lemma 2.3, we have $\left.\psi_{*}^{\mu} X(\mu)\right|_{\psi^{\mu}\left(p_{0}\right)} \neq 0$ and $\left.\psi_{*}^{\mu} X(\mu)\right|_{\psi^{\mu}\left(p_{i}\right)}=0$, but by condition $\psi^{\mu}\left(p_{i}\right) \neq p_{0}$, we know that $\left.\psi_{*}^{\mu} X(\mu)\right|_{p_{0}} \neq 0$, as in the proof of Theorem 3.1, we can prove that $J_{0}\left(p_{0}\right)=0$ and $J_{\mu}\left(p_{i}\right)=0(\mu \neq 0,1 \leq i \leq k)$.

COROLlaRY 3.3. Let $X(\mu)$ be a Lie rotated vector field, $\left.X(0)\right|_{p_{0}}=0$, if $\left.Y\right|_{p_{0}} \neq 0$, but for some $i_{0}\left(1 \leq i_{0} \leq k\right)$, set up $\psi^{\mu}\left(p_{i_{0}}\right)=p_{0}(\mu \neq 0)$, then $J_{0}\left(p_{0}\right)=j_{\mu}\left(p_{i_{0}}\right)$, $J_{\mu}\left(p_{i}\right)=0\left(\mu \neq 0,1 \leq i \leq k\right.$ and $\left.i \neq i_{0}\right)$.

EXAMPLE 3.4. Let $X(\mu)=\left(x_{2}^{2},-x_{1}+\mu\right)$, and let

$$
Y=\left(3 x_{1}-\alpha x_{2}, 2 x_{2}\right)
$$


when $|\mu|<\delta$, we take $\alpha>0$ and $\alpha \ll 1$, on the range of $D=\left\{\left(x_{1}, x_{2}\right) \mid x_{2}<\alpha^{-1}\right\} \subset \mathbb{R}^{2}$, at all ordinary points $\in D$ of $X(0)$, set up

$$
L(0)=\alpha x_{1}^{2}+x_{2}^{2}-\alpha x_{3}^{2}>0,
$$

that is, $X(\mu)$ constitutes a Lie rotated vector field on $D$, the singular points of $X(\mu)$ are strictly moved as parameter $\mu$ is changed. We note that $\left.Y\right|_{p_{0}}=0, p_{0}=(0,0)$ is singular point of $X(0)$, by Theorem 3.1, we can find that $J_{0}\left(p_{0}\right)=0$ and $J_{\mu}\left(p_{i}\right)=0(\mu \neq 0)$, where $p_{i}=(\mu, 0)$.

THEOREM 3.5. Let $X(\mu)$ be a Lie rotated vector field, $\left.X(0)\right|_{p_{0}}=0$, $p_{0}$ is elementary.

(1) If $\left.Y\right|_{p_{0}}=0$, then $p_{0}$ cannot be moved as parameter $\mu$ is changed.

(2) If $\left.Y\right|_{p_{0}} \neq 0$, then $p_{0}$ can be moved as parameter $\mu$ is changed, and the moved point is the singular point $\psi^{-\mu}\left(p_{0}\right)$ of $X(\mu)(\mu \neq 0)$.

Proof. (1) We note $J_{0}\left(p_{0}\right)= \pm 1 \neq 0$, it is proved immediately from Theorem 3.1.

(2) First of all, we prove that $p_{0}$ is indeed moved as $\mu$ is changed, suppose that it is not real, i.e., $p_{0}$ is not moved as $\mu$ is changed, then that $\left.X(\mu)\right|_{p_{0}}=0(\mu \neq 0)$, by Lemma 2.3, we know that $\left.\psi_{*}^{\mu} X(\mu)\right|_{\psi^{\mu}\left(p_{0}\right)}=0$. Because $p_{0}$ is isolate singular point of $X(0)$, we take $\bar{\delta}>0$ and ample small $\eta>0$, it follows that $\psi^{\mu}\left(p_{0}\right) \notin \overline{S_{\eta}\left(p_{0}\right)}$. When $0<|\mu|<\bar{\delta}<\delta$, then for $\partial S_{\eta}\left(p_{0}\right)$, we have $J_{\mu_{*}}\left(p_{0}\right)=0$ (since $\left.\psi_{*}^{\mu} X(\mu)\right|_{p_{0}} \neq 0$ ), where $\mu \neq 0$. But $J_{0}\left(p_{0}\right)= \pm 1 \neq 0$, this is a contradiction from Lemma 2.5. Thus we have proved $p_{0}$ is indeed moved as $\mu$ is changed, and by Corollaries 3.2 and 3.3, it follows that $p_{0}$ is moved as the singular point $\psi^{-\mu}\left(p_{0}\right)$ of $X(\mu)(\mu \neq 0)$ when $\mu$ is changed.

LEMMA 3.6. Let $X(\mu)$ be a Lie rotated vector field, $\left.X(0)\right|_{p_{0}}=0$, and there is an elliptic region at the singular point $p_{0}$.

(1) If $\left.Y\right|_{p_{0}}=0$, then the singular point $p_{0}$ cannot be moved when parameter $\mu \neq 0$.

(2) If $\left.Y\right|_{p_{0}} \neq 0$, then when parameter $\mu \neq 0$, singular point $p_{0}$ is moved, and $p_{0}$ be moved as singular point $\psi^{-\mu}\left(p_{0}\right)$ of $X(\mu)$.

Proof. (1) We already know that $\left.Y\right|_{p_{0}}$, suppose the original equation is not real, then when $\mu \neq 0$, singular point $p_{0}$ is moved, thus we let $p_{0}$ moved as the singular point $p_{\mu}$ of $X(\mu),\left.X(\mu)\right|_{p_{\mu}}=0, p_{\mu} \neq p_{0}, \mu \neq 0$. From Lemma 2.3, we know that $\left.\psi_{*}^{\mu} X(\mu)\right|_{\psi^{\mu}\left(p_{\mu}\right)}=0$, and by $\left.Y\right|_{p_{0}}=0$, we know that $\psi^{\mu}\left(p_{\mu}\right) \neq p_{0}(\mu \neq 0)$. Let $\Omega$ be an elliptic region at the singular point $p_{0}$ of $X(0)$, for arbitrary fixed $\mu(0<|\mu|<\delta)$, it is sure to have some elliptic trajectory $r$ of $X(0)$, which does not contain the point of $\psi^{\mu}\left(p_{\mu}\right)$ on $r$ and in $r$. Since $r$ has single direction, and there is no singular point of $\psi_{*}^{\mu} X(\mu)$ on $r$ and in $r$. By Lemma 2.5, we can know that positive half trajectory or negative half trajectory of $\psi_{*}^{\mu} X(\mu)$ which pass through the point $p$ will wander about without a home to go to, where $p$ is any point which passes the inner region of $r$, this is a contradiction.

(2) Now we know $\left.Y\right|_{p_{0}} \neq 0$, yet use reduction to absurdity. Suppose, when $\mu \neq 0$, singular point $p_{0}$ is not moved, i.e., establish $\left.X(\mu)\right|_{p_{0}}=0$, namely we have $\left.\psi_{*}^{\mu} X(\mu)\right|_{\psi^{\mu}\left(p_{\mu}\right)}=$ 0 , and $\psi^{\mu}\left(p_{0}\right) \neq p_{0}$. The method of the proof is completely alike as part (1), we can prove it is a contradiction. Thus let $\mu \neq 0$, singular point $p_{0}$ is moved as singular point $p_{\mu}\left(p_{0} \neq p_{\mu}\right)$ of $X(\mu)$, i.e., $\left.\psi_{*}^{\mu} X(\mu)\right|_{\psi^{\mu}\left(p_{\mu}\right)}=0(\mu \neq 0)$. If $\psi^{\mu}\left(p_{\mu}\right) \neq p_{0}$, the 
method of the proof is alike as in part (1), yet it is a contradiction, thus only establish $\psi^{\mu}\left(p_{\mu}\right)=p_{0}$, or $p_{\mu}=\psi^{-\mu}\left(p_{0}\right)$.

LEMMA 3.7. Let $X(\mu)$ be a Lie rotated vector field, $\left.X(0)\right|_{p_{0}}=0$, and when $\mu \neq 0, p_{0}$ is moved as the singular point $p_{\mu}\left(p_{\mu} \neq p_{0}\right)$ of $X(\mu)$ as $\mu$ is changed. If $\left.Y\right|_{p_{0}}=0$, then for singular point $p_{\mu}\left(\right.$ or $\left.p_{0}\right)$, at least there are a positive half trajectory and a negative half trajectory of $X(\mu)$ (or $X(0))$ to get into it.

Proof. We only prove the circumstance of point $p_{\mu}$ (the proof is completely alike as the circumstance of point $p_{0}$ ).

From Lemma 3.6, we know that there is no elliptic region which links with the singular point $p_{0}$ of $X(0)$, the same do the singular point $p_{\mu}$ of $X(\mu)$, and from Theorem 3.1, we know that the index of $p_{\mu}$ of $X(\mu)$ is zero. Take $p_{\mu}$ as circular center, make the circumference of a circle $l$ with radius rather small, and let that hyperbolic region of point $p_{\mu}$ which intersects with the circumference of a circle $l$ has $h$. By the Bendixson's formula in $\S 6$ of Chapter 3 of [8], we can immediately find $h=2$.

From Lemmas 3.6 and 3.7, we have the following theorem.

THEOREM 3.8. Let $X(\mu)$ be a Lie rotated vector field, $\left.X(0)\right|_{p_{0}}=0$, and let $\left.Y\right|_{p_{0}}=0$, then some singulars while can be moved as parameter $\mu$ is changed in $X(\mu)$ only contain two hyperbolic regions and their index is zero.

ACKNowledgement. The authors express grateful thanks to Professor Yaoxian Wang for his help and direction in the work.

\section{REFERENCES}

[1] W. M. Boothby, An Introduction to Differentiable Manifolds and Riemannian Geometry, Academic Press [A subsidiary of Harcourt Brace Jovanovich, Publishers], New York, London, 1975. Zbl 333.53001.

[2] S. S. Chen and W. H. Chen, Lectures in Differential Geometry, Beijing Univ. Press, Beijing, 1983.

[3] Y. Y. Chen, Limit cycles and quasirotational vector fields, Acta Math. Sinica 32 (1989), no. 6, 786-792. MR 91c:58113.

[4] G. F. D. Duff, Limit-cycles and rotated vector fields, Ann. of Math. (2) 57 (1953), 15-31. MR 14,751c. Zbl 050.09103.

[5] Z. E. Ma, The motion of singular closed orbit rotated vector fields, J. Xi'an Jiaotong Univ. 4 (1978), 49-65.

[6] L. M. Perko, Rotated vector fields and the global behavior of limit cycles for a class of quadratic systems in the plane, J. Differential Equations 18 (1975), 63-86. MR 51\#10752. Zbl 297.34024.

[7] Y. Q. Ye, S. L. Cai, L. S. Chen, K. C. Huang, D. J. Luo, Z. E. Ma, E. N. Wang, M. S. Wang, and X. A. Yang, Theory of Limit Cycles, American Mathematical Society, Providence, R.I., 1986. MR 88e:58080.

[8] Z. F. Zhang, T. R. Ding, W. Z. Huang, and Z. X. Dong, The Qualitative Theory of Differential Equation, Science Publishing House, Beijing, 1985.

Jie WANG AND Chen Chen: School of Electric Power, Shanghai JiAOtong University, SHANGHAI, 200030, CHINA 


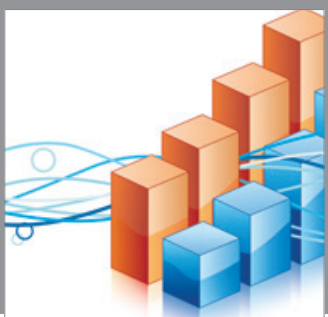

Advances in

Operations Research

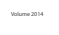

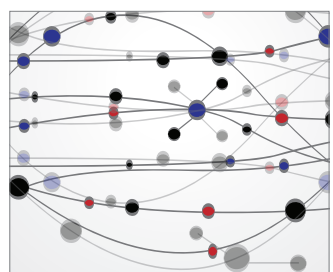

\section{The Scientific} World Journal
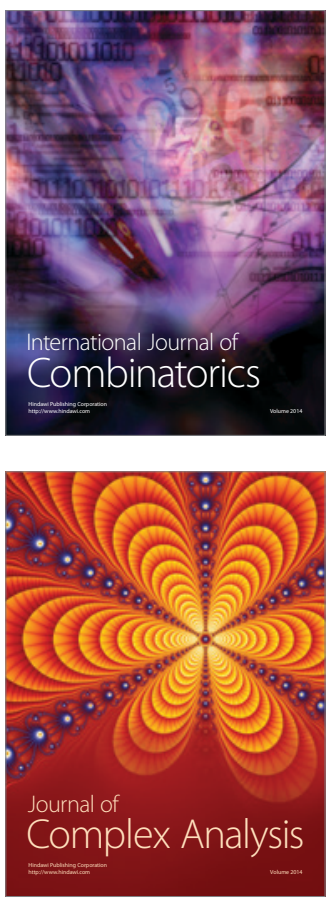

International Journal of

Mathematics and

Mathematical

Sciences
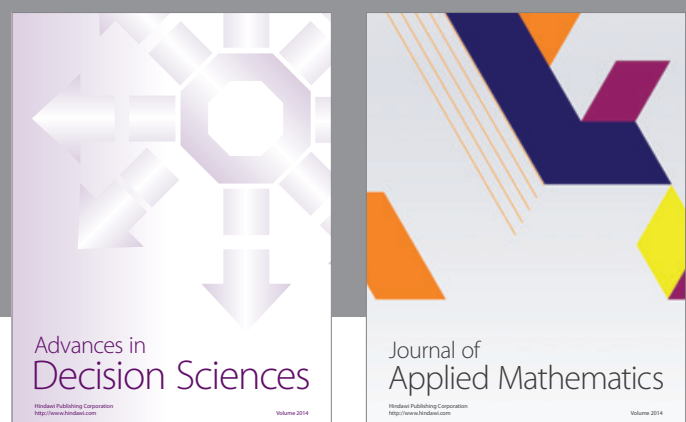

Journal of

Applied Mathematics
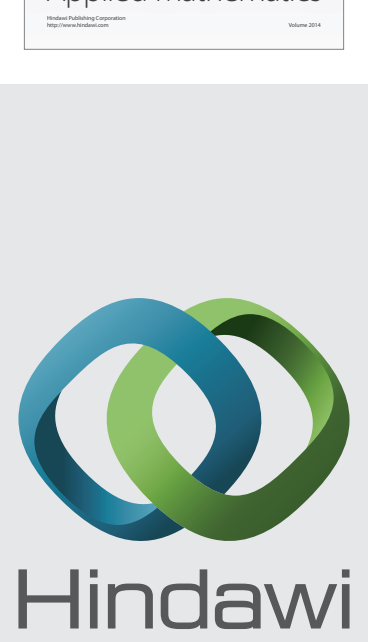

Submit your manuscripts at http://www.hindawi.com
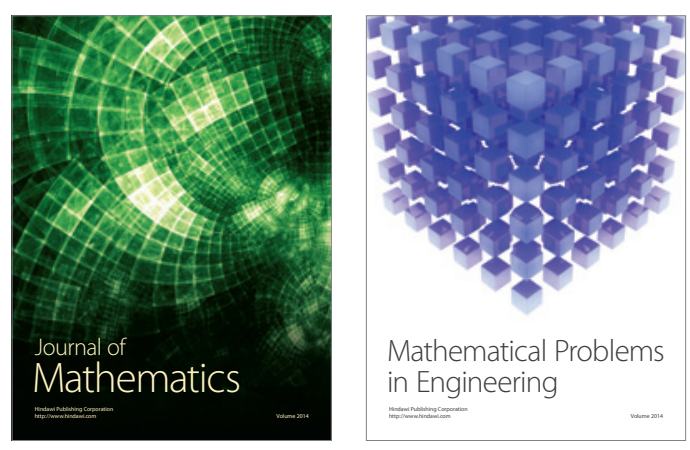

Mathematical Problems in Engineering
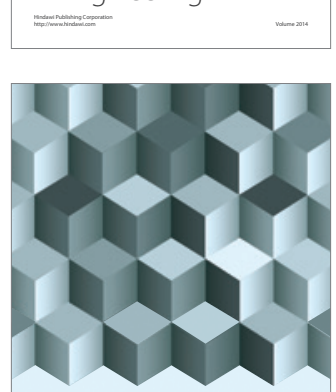

Journal of

Function Spaces
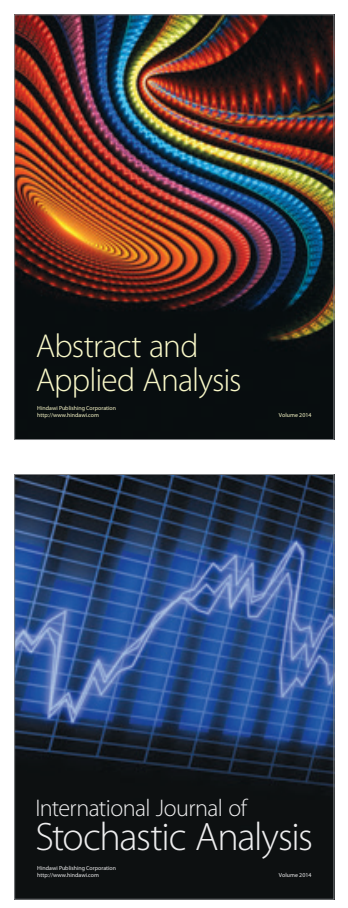

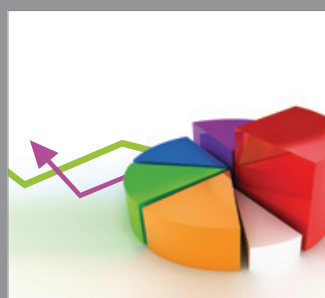

ournal of

Probability and Statistics

Promensencen
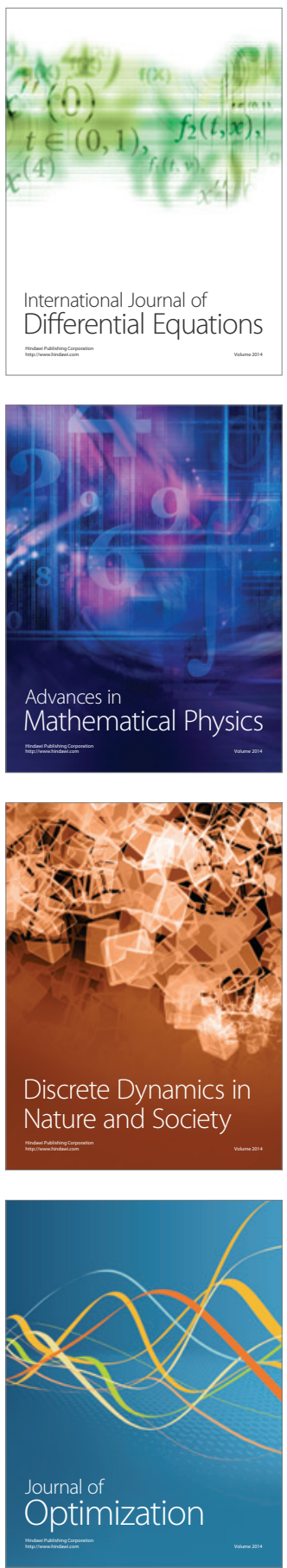